EPiC Series in Computing
Volume 69, 2020, Pages $80-89$
Proceedings of 35th International Confer-
ence on Computers and Their Applications

\title{
Computing Urban Fabric of a Historical Town
}

\author{
Naai-Jung Shih ${ }^{*}$, Cheng-Yu Hsieh, Yi Chen, and Pei-Huang Diao \\ National Taiwan University of Science and Technology, Taipei, Taiwan \\ shihnj@mail.ntust.edu.tw, fish8410@gmail.com, \\ m10713036@mail.ntust.edu.tw, diaoph@msn.cn
}

\begin{abstract}
Traditional computer simulation is replaced by 3D scans of the temporary urban fabric in a Taiwan heritage site for the quantitative assessment of local evolvement. The transfer of as-built point cloud model to the vertical and horizontal sections enables the inspections of evolved openness types on an old street enclosed by building facades and remodeled building components. Temporary fabrics, which consist of the installations and components, are represented in terms of the modification ratio on facade. The ratio contributes to the balance between the maintenance of cultural identity and the development of supporting commercial facilities made by local efforts. The variation changes along the old street cross districts of preservation, commercial and residential areas. Result shows the highest ratio exist in commercial district, where the highest ground activity along the entire street has created a typology of $\mathrm{T}$ or enclosed section of open space, as shown in point cloud model which is so realistic that no former computer models can display.
\end{abstract}

\section{Introduction}

Lukang, a heritage town in center Taiwan, has a unique architectural style and urban fabric from the earliest stages of its development (1683-1777), development peak (1784-1836), recession (18511888), degeneration (1895-1943), recovery (1945-1987), and current development phase (1988-now). It is located on the west coast of Taiwan and was formerly the second-largest harbor between the 17th and 19th centuries. Its competitive advantage disappeared after port silting and the rejection of railroad deployment [1]. The slowdown of local development and modernization eventually prevented the city's over-expansion and the potential deconstruction of historical heritages.

The distinguishing characteristics in its landscape, humanity, and styles, however, have been wellpreserved, making it one of the most important sightseeing locations in Taiwan. A large number of cultural relics exist around the Zhongshan Road, Zhongzheng Road, and peripheral regions, which are also known as the Old Streets. A Heritage Preservation District was established by past government

${ }^{*}$ Corresponding author 
promotion strategies. The old streets have developed into a unique block-based commercial pattern of cultural industry. For example, one linear old alley has connected historical blocks and created a rich tapestry of food, handcrafts, religions, and open spaces. The tourist routes also vary by subject. Threedimensional scans have been made to a famous old street over approximately $2500 \mathrm{~m}$ using Faro Focus $3 D^{\circledR}$ (Figure 1).

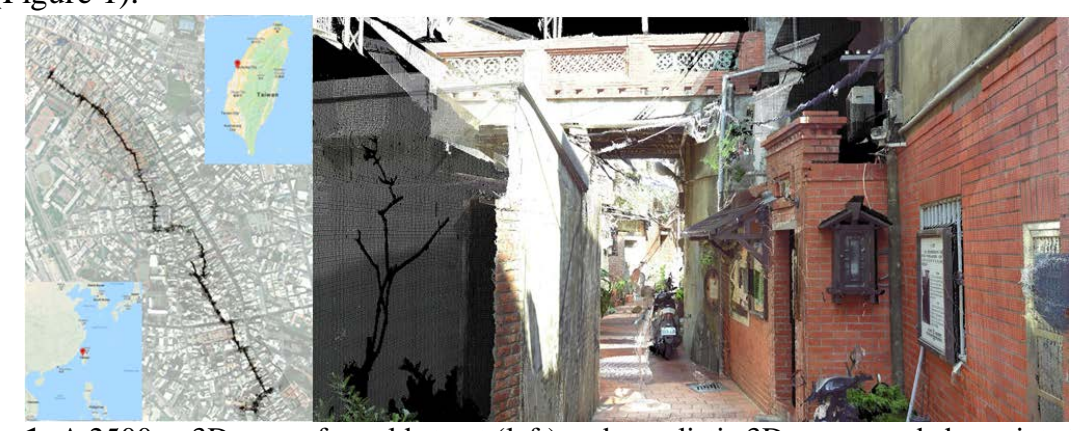

Figure 1: A $2500 \mathrm{~m}$ 3D scan of an old street (left) and a realistic 3D scene made by point cloud (right)

The alleys around Nine-turns Lane have created a famous sightseeing trail. The role of old residence has gradually transferred and remodeled to cafés, creative cultural souvenirs shops, or studios for hands-on experience. Transferring from residence to a shop requires the redesign of building front and interior. Signage, panels, or billboards have been progressively added to façade as a new type of identity. The old image and identity have gradually changed with characters carefully preserved to remind visitors of old days. Majority of wall materials, construction manners, and landscape can still be identified.

This paper is structured as follows. Section 1 introduces the background of the historical town. Section 2 explores current technology and its application in heritage study. Section 3 addresses the intention to quantify the auxiliary urban fabric outside building boundary. Section 4 specifies how 3D scan data were applied to illustrate the urban fabric and retrieval approach. Section 5 explains how the temporary settings were created as the micro expression of fabric. Section 6 estimates the modification ratios. Section 7 correlates the ratio and districts. Section 8 presents the conclusion of this paper.

\section{Related Works}

Three-dimensional (3D) scans have been used for data collection in large areas to create urban models. The point cloud was used to analyze urban open space, buildings, and landscapes [2] [3] [4] [5] [6]. The as-built information at full-scale provides detailed components with a high precision level of measurement. In contrast to drawings or maps that are not currently updated, 3D scans provide an efficient and effective on-site data collection approach for 3D configurations that are usually unavailable.

The study of a heritage site usually requires the creation of a 3D model for preservation or tourism purposes. The models are used for documentation, measurement, or illustration. The recorded contents not only illustrate the open space, but also the urban fabric that is made of buildings, culture entities, or the landscape. Photogrammetry technology has been widely applied in unmanned aerial vehicles (UAVs) or unmanned aircraft systems (UASs) [7] [8]. Four-dimensional dynamic scene reconstruction has been developed as an efficient modeling approach using videos or photos as input resources [9]. For the capture of detailed models, 3D scanning can create objects of the original scale (in 1:1) to fulfill measurement needs in architectural professional practice. An automatic scan process 
creates to a scene up to $360 \times 300$ degrees of an environment in a range of $100 \mathrm{~m}$ or more. The automatic registration of multiple scans is made, no matter whether it is a large site or a long alley. A point cloud model has been widely used for construction documentation.

Sustainable development in policy-making has profound implications for the practice and politics of urban and regional development [10]. A sustainable city can improve satisfaction with city life by offering many positive experiences and perspectives [11] [12] [13]. Culture and heritage are now widely perceived to be important contributors to a sustainable development [14]. The economic, environmental, and social components of sustainable development are interdependent and mutually reinforced [15]. Based on the historical changes of urban fabric around Old Street from 1683 to 2018, development on Old Street has been constantly occurring as part of the economic development. Considering the interdependence of the three sustainable components, economic development has to be connected with social development and environmental protection as well. The level of interdependence and mutual reinforcement, which may vary between cities, provides a trail of local history. All the modifications of fabric and second contour represent an attempt to sustainably evolve the culture-related spatial structure, developed and managed by local efforts and government promotions.

\section{Research goal}

A sustainable process of development should be correlated with today's urban fabric for what a town has been experienced. The analysis should present a digital bridge between history and local development, in terms of humanity and environment. The study evaluates the level of development by quantifying the auxiliary urban fabric outside building boundary in an old street space. In addition to the field observation, the estimation should be made based on the as-built measurement for a precise description of variations. A digital twin needs to be created for the cultural heritage site in the quantification process as a connection to urban zoning characteristics by building components on vertical and horizontal sections.

\section{Urban Fabric and Retrieval Approach}

Urban fabric stands for building enclosures that define open spaces. Different fabric relationships lead to a reconceptualization of urban data that are utilized to support the representation of detailed heritage configuration. The data are converted to desired forms as needed. This is particularly the case when studies include as-built scan data for a full-scale description of an environment. The retrieval of urban fabric usually comes from geographic maps in 2D. Recent studies have emphasized second contours (or second skins), which contribute a new, remodeled, and yet flexible existence of installations to the definition of urban fabric [16]. Two-dimensional maps or GIS model can no longer meet the demand in terms of descriptions for the geometries or surface attributes.

Urban fabric, which is determined by the projection of outer boundary, is made of fixed parts or temporary settings. Although the configuration is restricted by the site, the final appearance is usually changed after years of occupancy. One typical example is the cantilevers made by commercial signage, HVAC or mechanic facilities, or even illegal constructions to fulfill the daily needs of business or living. In general, the original fabric is static and should be consistent with what is shown on maps. Great diversity exists in reality. The fixed part of fabric was added by local residents as canopy or rain shield at entrance. The overhang canopy or rain shield changes the sky openness into full open, semi-open, or fully covered pattern. Similar pattern was either distributed interlaced to create a rhythm of light and shadow or connected in a continuous manner to make a street covered 
like a tunnel. The pattern of distribution created new and unique landscape which is now dramatically different from what a street should appear at original built stage.

The identify of adds-on was important and should be very sensitive to the type and shapes that were incompatible or controversial to existing style or vocabulary. The measurement of individual entity was almost impossible and very effort-consuming. In order to increase the efficiency and to maintain the correctness, 3D scans were applied to solve the problems with effectiveness and efficiency. The detail level of the as-built data enables a unique perspective in defining fabric types, relationships, and operations that are applied to facilitate the quantitative studies. The researchers can work with the cloud data afterward in the laboratory and conduct inspection of shape or size away from the site. Comparing to the time spent on field scan, the off-site data manipulation has been increased many times from a few days to all four months in an academic semester.

In contrast to the traditional application of 3D scan in the preservation of heritage site with visual emphasis, this study took a deep investigation of the 3D point cloud data to explore the possible evidence of local development. The instances usually limit to building front. However, the collection of all instances along an alley or a street creates a distinguishing image and local identity, which is not only recognized by residences, but also attracts tourists as part of cultural tourism. The size of modification does not have to be a major remodeling of an entire block or the reconstruction of a whole building. The sign of changes may occur to the peripheral boundary of private property. Some of them may intrude public space for personal use.

Satellite images are inspected individually and overlaid from 2002 to 2018 (Figure 2). The inspection is made by showing the difference on map for horizontal fabric. The result shows occasional occurrences of changes that were either added or removed next to building boundaries. The evolvement of fabric is a periodical and chronological process with most of the changes occurred on commercial district.

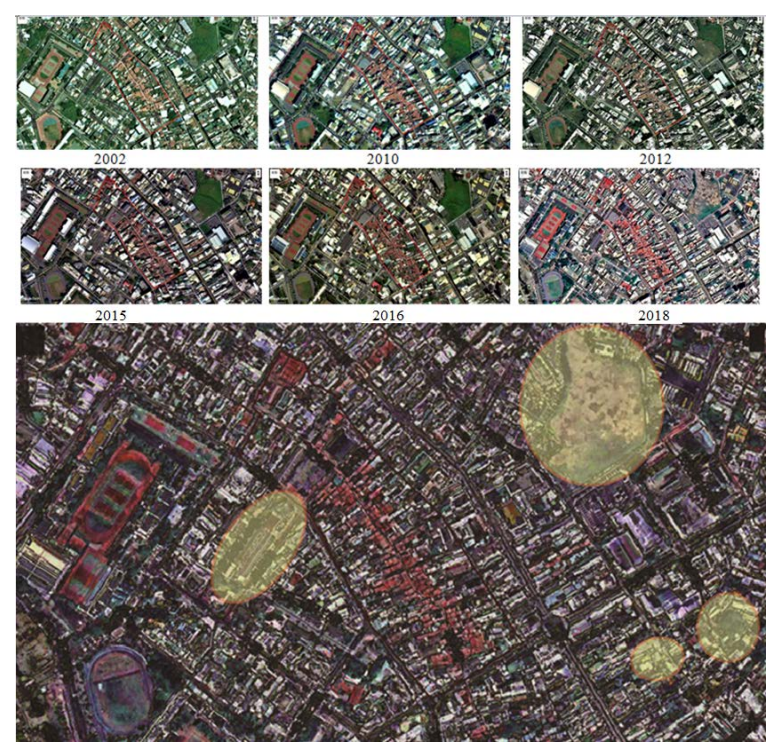

Figure 2: Satellite image-based fabric differentiation between 2002 and 2018

\section{The Micro Expression of Fabric}

The installations added to building enclosure fulfill functional needs in a permanent or temporary manner (Figure 3 \& Figure 4). The dynamic deployment creates micro expression with features 
varying from retractable weather shield, mobile booth, to lighting fixtures. The expression is different by weather, daylight, or events. The expression is similar to 3D graffiti that is shop-dependent or section-dependent. In other words, the expression next to a temple or a market is different from that next to a residence area. The pattern of distribution has formed local identity that attracts visitors by following a tourism route that connects the locations of modified expressions.

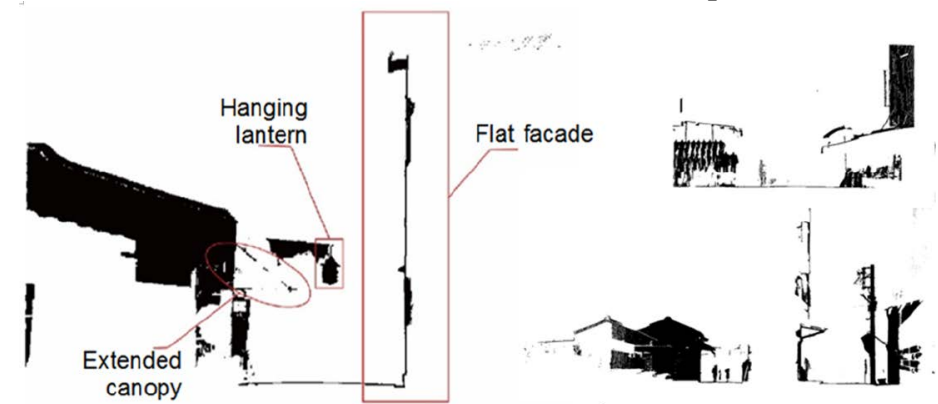

Figure 3: The micro expression of a building (left) and the typical type of weather shield in residential, commercial, and preservation district (right)

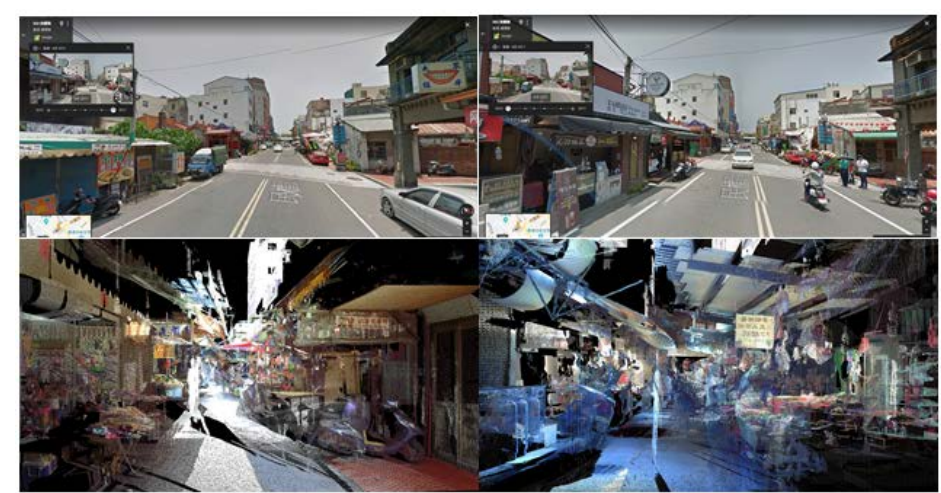

Figure 4: The difference on façade made by commercial activities on 2009 (top left) and 2017 (top right); the resulting complexity of fabric occurs in or next to a traditional market (bottom)

The temporary settings or installations, which were usually not considered as part of urban fabric, did change the projected configuration of building boundary. The settings are made by property owners, local business associations, or government tourism department. Private owners may have installations extruding into public space for business purpose. The open space, like a street, is decorated by installations during festivals to promote cultural activities by local associations or government department. The temporary settings may only last for a few months. The configuration of a space was changed accordingly during this period of time and created a new meaning to local identity. The promotion of local cultural tourism or even the private modification to building front has close connection to the temporary fabric and deserves a study from a quantitative point of view. The quantitative measurement, as an assessment of its influence, serves as a variable that defines the most update development of a historical alley.

The emerging of urban fabric is either a long-lasting evolving process or a short event. The former refers to the casual or individual building remodeling by private owners or the government-conducted urban renewal. The latter refers to the fairs or events occurred monthly, seasonally, annually, or at a specific time period.

A 3D point cloud model is feasible to present the configuration as a whole. The physical layout of fabric is shown in vertical and horizontal manner. The fabric reveals different characteristics of 
geometries that contribute to cultural icons, identities, temporary installations, permanent facilities, and building boundaries. The layout is permanently or temporarily installed to reconstruct a space which leads to the identity of a city or its development.

- The horizontal fabric varies by levels of height: A defined thickness of cloud is sliced to reveal the horizontal section of a space within a defined boundary. The fabric, which bears similar configuration from ground level to the roof level (Figure 5), defines the boundary between interiors and exteriors. Variations occur after decades of development or remodeling.

- The vertical fabric varies by thickness and distance: A defined thickness of cloud is sliced to reveal the vertical section of a space at certain location or orientation. The span varies from the width of a small alley to an entire town with the focused region extended to a urban scale.
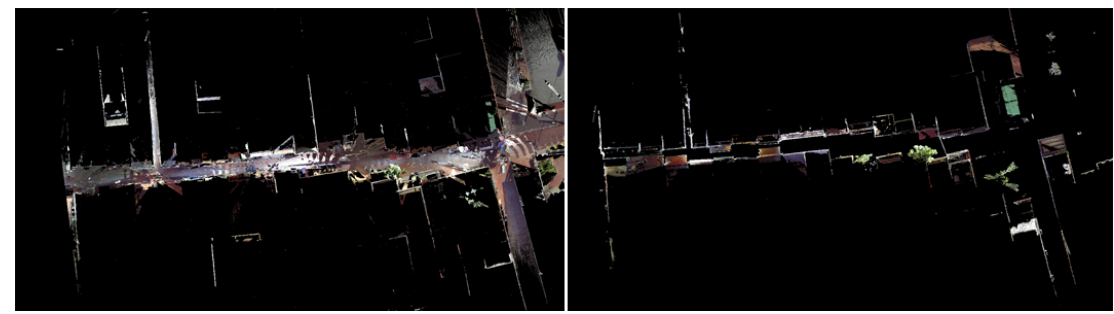

Figure 5: The horizontal fabric varies by levels of height

Both the vertical and horizontal fabrics are different by streets. In order to highlight the changes, vertical sections are overlaid by referring to façade or street central line. The former refers to only one side of the street facades. The latter, which is based on the central line, can illustrate the different façade setback distance on either side, the density of activity on the street, and the openness above street.

The union image of profiles quickly characterizes a street by $\mathrm{U}$, 凸, $\mathrm{T}$, $\square$, and L types (Figure 6). The overlaid images contribute to the comprehensive description of static subjects, such as buildings, advertisement panels, power wires, or HVAC facilities, plus dynamic subjects, such as tree configurations, pedestrians, vehicles, or mobile booths. The final image represents a location-specific character of the proportion to human scale, the composition of subjects, and the distribution of activities.
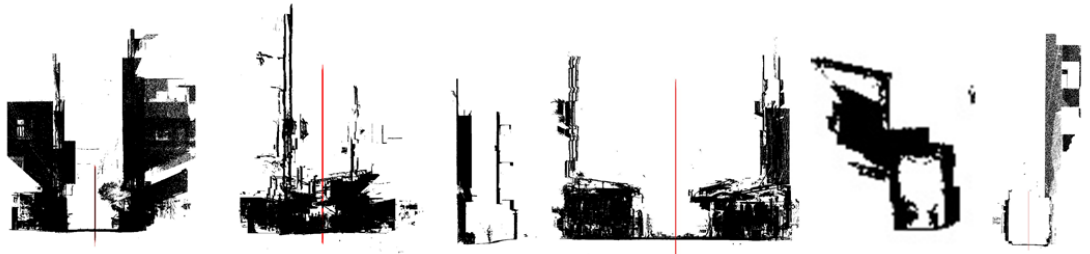

Figure 6: The profiles in U, 凸, T, 口, and L shapes

The five types of profiles represent an evolving process of local history. The original urban planning started since Japanese colonial period in Taiwan. The difference between " $U$ ” and “凸” types is the space designed to invite pedestrians on ground level in front of a building with recessed and secured enclosure. After years' development of commercial district, the " $U$ " type has become " $T$ " type with space extended toward street center under rain shield on ground level. Most part of the covered-up streets in “ם" type was remodeled intensively. As a result, the old urban image, so-called covered-up sky and ground, was changed into new urbanscape located next to the old houses in preservation district. 


\section{Estimation of the Modification Ratios}

This study started from identifying the reasons and specific issues related to the local remodeling efforts on building front and façade. The process is followed by the selection of building components that are closely connected to local activities. The area of components was estimated to define their boundaries of influences and possible intensities of impact by projections. The results are the modification diagram that illustrate the shift of characteristics in a historical alley from north to south.

The recognition of property-based fabric definition is blurred in reality. The horizontal fabric is a collection of fuzzy boundary sets that come from the original building enclosure with temporary or permanent installations. The projection was made above $1.5 \mathrm{~m}$ and extended above streets in the vertical fabric, including trees planted by local residents (Figure 7). The representation is made of building boundary in solid line and canopies in dot line (Figure 8).

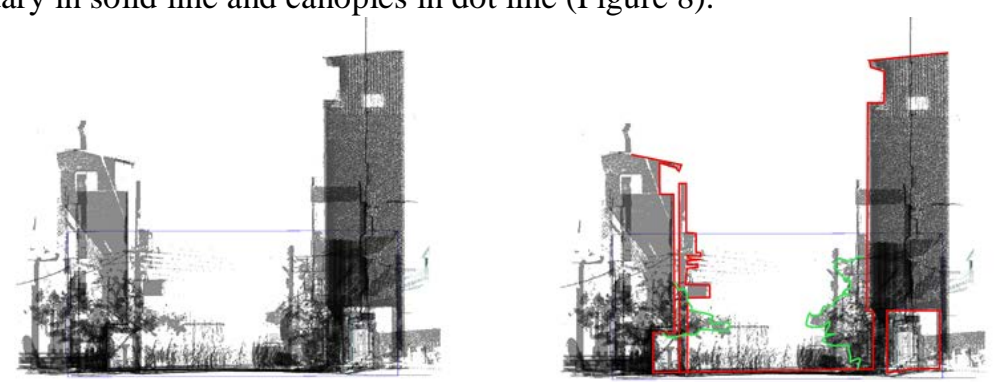

Figure 7: The overlaid sections made by point cloud (left) and boundary in vector drawings (right)
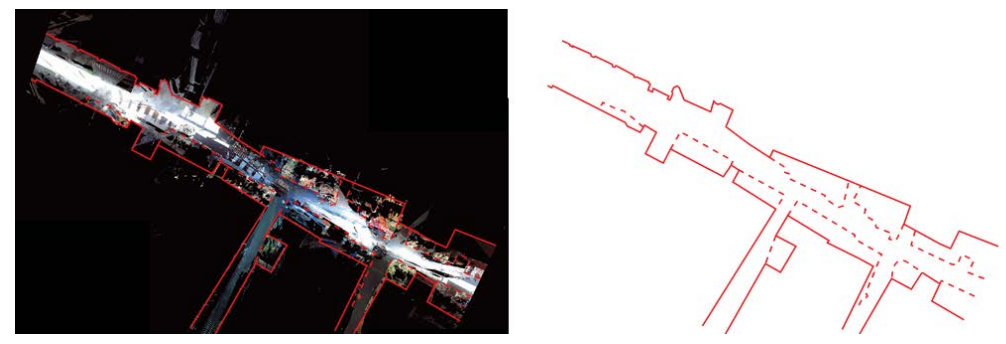

Figure 8: The difference between original building boundaries and extended ones (above) and the significant difference occurs to the façade on either side of a street (bottom)

The outline of projected canopy area illustrates a specific distribution pattern in each segment. The interleaved canopies indicate where shops are located and how the pattern is shifted on both sides of the alley. A shifted allocation would present a lower density of commercial activity, comparing to other segments where both the canopies and mobile booths are connected shop by shop. The facilities in commercial district, which are extended from interiors to shop front, significantly narrow down the street width and eventually make it a pedestrian area. This situation occurs near traditional market which not only service local people with daily needs, but also attracts tourists for the reminiscence of old day's shopping experience. It seems the gap between two walking people is much shorter in this commercial segment.

Temporary fabrics, which consist of the installations and components, are represented in terms of the modification ratio on façade: the ratio is calculated by dividing the projected area of additive building parts by the entire projected façade area (Figure 9). The ratio, which illustrates the amount of modification that is perceived along the small alley, is an index of adaption from local residences within the incremented space to meet living or commercial needs in these days. The newly added part, which is called the $2^{\text {nd }}$ contour, is usually made of remodeling or illegal constructions and does not 
belong to the original design. The modified part may not be consistent with the original building vocabulary of cultural heritage. In general, the larger the ratio is, the more deviation was made to the original alley scene in the old days.

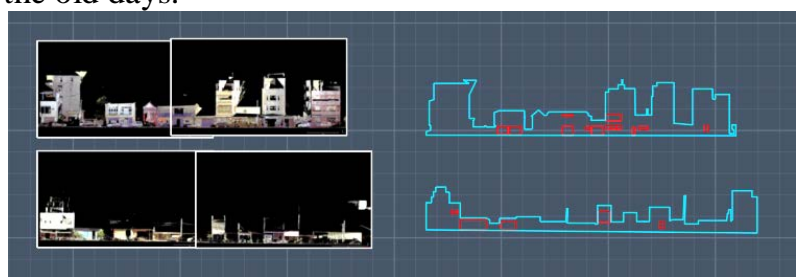

Figure 9: Modification ratio is made by defining the modified regions and conducting area estimation

\section{Discussion}

The development of the Old Street revealed a sustainable development process that was subject to economic recovery and government promotion projects. The spatial structure remains steady, however the $2^{\text {nd }}$ contour is constantly evolving. With concern about the environment, the development process now involves creating spaces for more sustainable programming of local culture promotion, education, or exhibition, by means of galleries, DIY workshops, or classrooms.

The ratio shows commercial district features the highest difference between the $1^{\text {st }}$ and the $2^{\text {nd }}$ contour. The remodeling was to attract customers. New space is also created to accommodate more people. Canopies that almost extended to the middle of street not only act as weather shield in a raining or sunny day, but also create a space in shop front to promote business. Years development of commercial activities have the " $U$ " type section of fabric become "T" or “凸" type with space extended toward street center on ground level. In contrast, residential area has higher expectation of living quality and prefers less interference of tourists. Only private parking space and landscape are featured instead.

Figure 10 shows the percentage of the $2^{\text {nd }}$ contour varies by streets and districts. The residential districts have the least percentage, in contrast to the commercial districts near traditional market with the highest ratio. The development of the contour is faster and more diversified than that in residential and preservation districts, where the temporary urban fabric presents similar character.

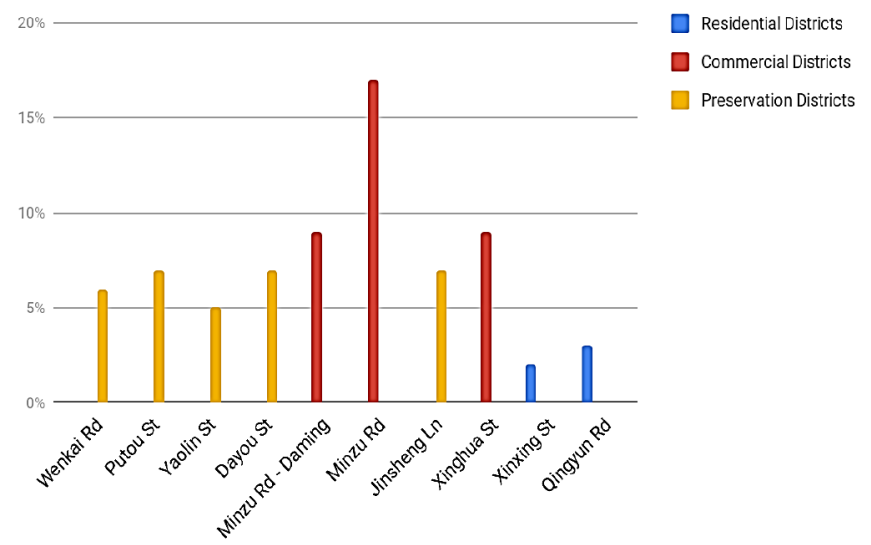

Figure 10: The variation of modification ratio by streets

The second contour of Old Street was programmed, designed, and evolved with local characteristics under the goal of creative management of commercial space. The shop signs and sun 
shades created the symbolic structure of the contour and enhanced the walking experience. The modified boundary of perception produced a distinct commercial image that extends local culture from the cultural creative industry to sustainable tourism.

The Old Street acts like a pathway behind the houses with long and narrow configuration. In contrast to the main entrances that usually face larger streets, the historical lanes or alleys are framed and isolated inside several street blocks as a hideaway from traffic and noise. In addition to the old layout, the Old Street has housed a number of artists dedicated to the preservation or creation of traditional arts in modern times, with hands-on experiences provided by workshops. Old Street has joined the surrounding tourism sites to form an enlarged cultural region of identity. A pavement system was also designed to connect Old Street and other sites under a government promotion project.

\section{Conclusion}

This study quantitatively defined the changes made to a heritage site based on the projected area of modifications made to façade as the $2^{\text {nd }}$ contour and the extended facilities over public street space as canopies and mobile booths. The estimation was conducted based on a precise 3D scan of an alley. The result shows the shops inside or next to traditional market or religion site, which create typical local scenes, have the highest ratio. The ratio variation is zoning-dependent.

The environment dimension in sustainable development remains to be explored. The recovery from an obsolete harbor attracted limited remodeling and new construction projects, and fewer investments from local residents or outside enterprises. Some shops along Old Street are rented from local residents. The new shops, with new business models, may gradually diminish the culture industry to a more commercially-oriented pattern. Whether the evolving model interacts with the social dimension remains to be seen. The three dimensions in sustainable development will be mutually reinforced into a Lukang-specific pattern. Old Street, which was sustainably transformed using much cleaner economic activities, brought wealth to the community and preserved the traditional culture. Cultural tourism, as a successful complimentary economic activity, has been developed with rich programming of spaces and with more environmentally-friendly awareness and technologies.

\section{Acknowledgment}

This research is sponsored by Ministry of Science and Technology of Taiwan. The involved project number is MOST 107-2221-E-011-029-MY3 (the second year). The authors express sincere appreciation.

\section{References}

[1] Y.-J. Wang, C. Wu and J. Yuan, "Exploring visitors' experiences and intention to revisit a heritage destination: The case for Lukang, Taiwan," J. Qual. Assur. Hosp. Tour., vol. 11, p. 162-178, 2010.

[2] N.-J. Shih and P.-H. Diao, "The preservation of railroad-related cultural activities and subjects using 3d scans and photogrammetry," Comput. Aided Design Appl., vol. 15, p. 129-137, 2018.

[3] A. Chase, D. Chase, J. Weishampel, J. Drake, R. Shrestha, K. Slatton, J. Awe and W. Carter, 
"Airborne LiDAR, archaeology, and the ancient Maya landscape at Caracol, Belize," $J$. Archaeol. Sci., vol. 38, p. 387-398, 2011.

[4] W. Zhou and A. Troy, "An object-oriented approach for analysing and characterizing urban landscape at the parcel level," Int. J. Remote Sens., vol. 29, p. 3119-3135, 2008.

[5] D. Evans, R. Fletcher, C. Pottier, J.-B. Chevance, D. Soutif, B. Tan, S. Im, D. Ea, T. Tin, S. Kim and e. al., "Uncovering archaeological landscapes at Angkor using lidar," in Proc. Natl. Acad. Sci. 2013, 2013.

[6] N.-J. Shih and C.-Y. Lin, "The evolving urban fabric and contour of old mountain street in Taiwan," Tourism Geogr., vol. 21, p. 24-53, 2019.

[7] F. Nex and F. Remondino, "UAV for 3D mapping applications: A review," Appl. Geomat., vol. 6, p. 1-15, 2014.

[8] I. Colomina and P. Molina, "Unmanned aerial systems for photogrammetry and remote sensing: A review," ISPRS J. Photogramm. Remote Sens., vol. 92, p. 79-97, 2014 .

[9] A. Mustafa, H. Kim, J. Guillemaut and A. Hilton, "Temporally coherent 4D reconstruction of complex dynamic scenes," in Proceedings of the IEEE Computer Society Conference on Computer Vision and Pattern Recognition, Guildford, UK, 26 June-1 July 2016.

[10] A. While, A. Jonas and D. Gibbs, "From sustainable development to carbon control: Eco-state estructuring and the politics of urban and regional development," Trans. Inst. Br. Geogr., vol. 35, p. 76-93, 2010.

[11] A. Beer, "Urban greenspace and sustainability," in Proceedings of the PRO/ECE-Workshop on Sustainable Urban Development: Research and Experiments, Dordrecht, The Netherlands, November 1993.

[12] Y. Jabareen, "Sustainable Urban Forms: Their Typologies, Models, and Concepts," J. Plan. Educ. Res., vol. 26, p. 38-52, 2006.

[13] A. Chiesura, "The role of urban parks for the sustainable city," Landsc. Urban Plan., vol. 68, p. 129-138, 2004.

[14] G. Brooks, "Heritage as a driver for development: Its contribution to sustainable tourism in contemporary society," in In Proceedings of the ICOMOS 17th General Assembly, Paris, France, 26 November-2 December 2011.

[15] UNIDO, "The Three Dimensions: Defining sustainable development," [Online]. Available: https://www.unido.org/sites/default/files/2015-

12/GC_16_7_Reissued_E_Implementation_of_the_Lima_declaration_Towards_ISID_V15073 01_0.pdf. [Accessed 15 July 2019].

[16] A. Yoshinobu, The Aesthetic Townscape, Tianjin, China: Baihua literature and art publishing house, 2006. 